\title{
High Resolution Melting Curve Analysis for Rapid Detection of Pyrazinamide Resistance in Mycobacterium tuberculosis Clinical Isolates
}

\author{
Fatima Osman', Farzana Ismail 1,2, Ayman Osman³, Shaheed Omar, Halima Said ${ }^{4}$, \\ Nazir Ismail4 \\ ${ }^{1}$ Department of Medical Microbiology, Faculty of Health Science, University of Pretoria, Pretoria, South Africa \\ ${ }^{2}$ National Health Laboratory Service, Tshwane Academic Division, Pretoria, South Africa \\ ${ }^{3}$ Department of Immunology, Faculty of Health Science, University of Pretoria, Pretoria, South Africa \\ ${ }^{4}$ Centre for Tuberculosis, National Institute for Communicable Diseases, Johannesburg, South Africa \\ Email: aymangassim@gmail.com
}

How to cite this paper: Osman, F., Ismail, F., Osman, A., Omar, S., Said, H. and Ismail, N. (2016) High Resolution Melting Curve Analysis for Rapid Detection of Pyrazinamide Resistance in Mycobacterium tuberculosis Clinical Isolates. Journal of Tuberculosis Research, 4, 155-172. http://dx.doi.org/10.4236/jtr.2016.44019

Received: May 26, 2016

Accepted: November 27, 2016

Published: November 30, 2016

Copyright $\odot 2016$ by authors and Scientific Research Publishing Inc. This work is licensed under the Creative Commons Attribution International License (CC BY 4.0).

http://creativecommons.org/licenses/by/4.0/ (c) (i) Open Access

\section{Abstract}

Background: Pyrazinamide (PZA) is one of the most important drugs for tuberculosis (TB) treatment, however, its susceptibility is not routinely tested. High-resolution melting (HRM) curve analysis has been widely used for many applications. In this study, HRM assay was developed and evaluated for the detection of PZA resistance in Mycobacterium tuberculosis clinical isolates. Methods: Ninety five M. tuberculosis clinical isolates with different susceptibility patterns to anti-TB drugs were used to evaluate this assay. Isolates were phenotypically (Bactec MGIT 960) and genotypically (HRM and pncA gene sequencing) analysed for PZA resistance. Results: Bactec MGIT 960 analysis revealed that 29 of the $95 M$. tuberculosis isolates were PZA resistant. In comparison to the Bactec MGIT 960, HRM showed a sensitivity of $47.7 \%$ and specificity of $74.6 \%$, and the overall agreement between the two methods was $68.4 \%$. Based on DNA sequencing, a correlation of 0.67 (significant at $p$-value $<0.05$ ) between phenotypic resistance to PZA and pncA mutations was observed. PZA resistance was strongly associated with multi-drug resistant (MDR)-TB as it was shown in $79.3 \%$ of the MDR isolates included in the study. Conclusion: HRM is simple and useful for screening clinical M. tuberculosis isolates for PZA resistance, however, further modifications to improve its performance are required.

\section{Keywords}

Pyrazinamide, Tuberculosis, High Resolution Melting Curve Analysis, Drug Resistance, pncA Gene 


\section{Background}

Pyrazinamide (PZA) is one of the most important drugs for tuberculosis (TB) treatment. It has a unique sterilizing effect on killing semi-dormant bacilli and is also very effective against multi-drug (MDR)-TB [1] [2]. Pyrozinoic acid (POA) is the active form of the drug and is metabolized by pyrazinamidase (PZase) enzyme which is encoded by the pncA gene [3]. Although number of studies suggest that PZA resistance is not caused by a single mechanism, mutations in the pncA gene are considered as the main mechanism of PZA resistance in M. tuberculosis [4]. The lack of reliable culture-based methods which require an acidic condition and high cost of the molecular techniques makes the testing of PZA resistance generally very difficult to perform [5]. It is very important to develop rapid and accurate drug susceptibility testing (DST) to prevent the spread of multi-drug resistant (MDR)-TB as well as extremely drug resistant (XDR)-TB.

Several molecular techniques have been described for the detection of anti-TB drug resistance-associated mutations, including the line probe assays GenoType MTBDRplus (Hain Lifescience GmbH, Nehren, Germany), GenoType MTBDRsl (Hain Lifescience $\mathrm{GmbH}$, Nehren, Germany), INNO-LiPA Rif.TB (Innogenetics, Ghent, Belgium), and Xpert MTB/RIF (Cepheid, Sunnyvale, CA) [6] [7]. However, these are probe based assays and only suitable for mutations that are located in hotspot regions of specific genes. Mutations in the pncA are dispersed throughout the gene and its upstream promoter; this makes the development of probe-based methods challenging [8]. Direct sequencing of pncA amplicons remains the best genotypic strategy, however this is costly.

High-resolution melting (HRM) curve analysis is a simple technique and has been widely used for many applications. PCR amplicons are heated and fluorescence loss is monitored in real-time. Variations in sequences are detected by difference in melting point $(\mathrm{T} m)$ compared to reference DNA; it does not require the use specific probes [9] [10]. In the TB field, HRM has been used for detecting rifampin (RIF), isoniazid (INH), streptomycin (STR), and fluoroquinolone resistant $M$. tuberculosis [11]. In this study, we describe an HRM technique to detect pncA mutations in $M$. tuberculosis clinical isolates and compare its results to the phenotypic PZA susceptibility testing (The Bactec MGIT 960). Isolates that had discordant results were sent for sequencing. This method uses the Roche LightCycler $480^{\circ}$ and involves simultaneous amplification of three overlapping fragments.

\section{Methods}

The study was conducted at the NHLS-TB Laboratory Tshwane Academic Division (Diagnostic division of the Department of Medical Microbiology, University of Pretoria from July 2013 to October 2013. Ethics approval was obtained (number 57/2012) prior to commencement of the study. The study isolates (95) were a mixture of MDR-TB and non-MDR-TB collected from the National Health Laboratory Service (NHLS) TB Laboratory. All isolates were presumptively identified as $M$. tuberculosis complex using Ziehl-Neelsen (ZN) stain and tested for susceptibility using the GenoType MTBDR plus 
line probe assay (HainLifescience $\mathrm{GmbH}$, Germany). Isolates were then categorized as either MDRs or Non-MDRs (Mono-RIF resistance, Mono-INH resistance or fully sensitive) [7].

Purity of all isolates by ZN staining was confirmed before susceptibility testing was performed. To exclude bacterial and fungal contamination, another amount of a wellmixed suspension was inoculated using sterile glass pipette on chocolate agar (produced in-house, NHLS Laboratory, Tshwane Academic Division) and incubated aerobically at $37^{\circ} \mathrm{C}$. All plates were inspected for bacterial growth every $24 \mathrm{hrs}$ for two days.

Isolates were sub-cultured onto 7H10 agar medium (Becton Dickinson, Sparks, MD, USA) and incubated for three weeks aerobically at $37^{\circ} \mathrm{C}$ until typical M. tuberculosis colonies appeared (dry, wrinkled, warty, with colourless rough surface). Colonies were picked and sub-cultured into MGIT tubes (Becton Dickinson, Sparks, MD, USA) according to the manufacturer's procedure.

Each isolate was tested for PZA susceptibility when the Bactec MGIT 960 indicated positive culture growth. The day the Bactec MGIT 960 (Becton Dickinson, Sparks, MD, USA) gives a positive signal is considered as day zero. Once positive, tubes were removed from the Bactec MGIT 960 (Becton Dickinson, Sparks, MD, USA) they were transferred into an incubator $37^{\circ} \mathrm{C} \pm 11^{\circ} \mathrm{C}$ until susceptibility testing was performed (within five days). Undiluted inoculum was used if the isolate was processed on days one or two. On days three, four or five, the growth culture was diluted 1:5 using normal saline (0.9\%) (SABAX Pour Saline, Adcock Ingram, South Africa) prior to susceptibility testing. PZA susceptibility testing was performed according to the manufacturer's procedure (Becton Dickinson, Sparks, MD, USA). Susceptibility results were available within four to 21 days. Once the test was completed, a report was generated and printed. Each isolate was categorized as " $\mathrm{S}$ " (susceptible) or "R" (resistant).

\subsection{DNA Extraction of M. tuberculosis Isolates}

Genomic DNA was extracted from the M. tuberculosis isolates using QIAquick PCR Purification Kit (Whitehead Scientific, Brackenfell, South Africa) according to the manufacture's procedure. Extracted DNA was quantified using specific spectrophotometry (NanoDrop; ND-1000; USA), and adjusted to a concentration of five to 30 $\mathrm{ng} / \mu \mathrm{l}$ using elution buffer (BioMérieux, France), this range is required by HRM for optimal performance. The extracted DNA was then transferred into a sterile $1.5 \mathrm{ml}$ tube (BioMérieux, France) and used directly for HRM analysis or stored at $-80^{\circ} \mathrm{C}$.

\subsection{HRM Optimization}

The LightCycler 480 HRM master mix contains all the ingredients required. The two most important contents which needed to be optimized carefully before commencing with the testing process were $\mathrm{MgCl}_{2}$ and primers.

\subsection{1. $\mathrm{MgCl}_{2}$}

Because specific amplification is essential for HRM analysis, determination of $\mathrm{MgCl}_{2}$ 
optimum concentration for each new primer pair is very important. A separate $25 \mathrm{mM}$ $\mathrm{MgCl}_{2}$ stock solution was supplied with the master mix. This is to allow the easy optimization of $\mathrm{Mg}^{2+}$ concentration. Optimization was achieved by running a positive sample with serial dilutions of $\mathrm{MgCl}_{2}(1.0,1.5,2.0,2.5,3.03 .5 \mathrm{mM})$ and analysis of the PCR products by agarose gel electrophoresis.

\subsubsection{Primers}

Primers previously described by Scorpio et al. were synthesized by Inqaba Biotechnical Industries Pretoria, South Africa to the M. tuberculosis pncA sequence (GenBank accession number U59967) [12] (Table 1). Prior to the experiment and to ensure specific amplification for HRM analysis, the optimal primer concentration (the lowest concentration that still results in a high rate of amplicon yield with a low cut off point $(\mathrm{Cp})$ and adequate fluorescence dynamics for a given target concentration) was determined. This was achieved by testing different concentrations of each primer $(0.1,0.2$ and 0.3 $\mu \mathrm{M})$.

\subsection{Real-Time PCR and HRM Conditions}

HRM curve analysis including the amplification step was performed using the LightCycler 480 High Resolution Melting Master Kit and the LightCycler 480 Instrument II (Roche, Germany). In order to detect all possible mutations in the pncA gene (561 bp) regardless of their locations, and to achieve the recommended size of the gene for HRM analysis (less than $300 \mathrm{bp}$ ), the target gene was amplified using three sets of primers to produce three fragments of the target gene [12].

The reaction mixture was prepared according to the manufacturer's instructions (LightCycler $480^{\circ}$ Real-Time PCR manual, 2009). HRM master mix consisted of $2 \times$ a concentration hot-start reaction mix that contained FastStartTaq DNA polymerase, reaction buffer, dNTP mix (with dUTP) and HRM Dye. PCR reaction composition is shown in Table 2. Inclusion of HRM dye enabled detection of double-stranded DNA by fluorescence, monitoring formation of amplicon during PCR cycling and melting curve analysis.

These components were added into a $1.5 \mathrm{ml}$ reaction tube on ice and mixed carefully by pipetting up and down. The amount in the volume column was multiplied by the

Table 1. Primers sequences of used for the amplification of pncA gene.

\begin{tabular}{|c|c|c|}
\hline \multicolumn{2}{|c|}{ Primers } & \multirow{2}{*}{$\begin{array}{c}\text { Sequences } \mathbf{5}^{\prime} \cdot \ldots \ldots \ldots \ldots . . . .3 \\
\text { F: GTCGGTCATGTTCGCGATCG }\end{array}$} \\
\hline \multirow[b]{2}{*}{ Set 1} & $\mathrm{P} 1$ & \\
\hline & $\mathrm{P} 2$ & R: TCGGCCAGGTAGTCGCTGAT \\
\hline \multirow{2}{*}{ Set 2} & P3 & F: ATCAGCGACTACCTGGCCGA \\
\hline & $\mathrm{P} 4$ & R: GATTGCCGACGTGTCCAGAC \\
\hline \multirow{2}{*}{ Set 3} & P5 & F: CCACCGATCATTGTGTGCGC \\
\hline & P6 & R: GCTTTGCGGCGAGCGCTCCA \\
\hline
\end{tabular}


Table 2. Reaction mixture used in HRM analysis for the detection of pncA gene mutation in $M$. tuberculosis.

\begin{tabular}{ccc}
\hline Reagents & Volume $(\mu \mathrm{l}) \times 1$ & Final Concentration \\
\hline Master Mix, $2 \times$ conc. & $10 \mu \mathrm{l}$ & $1 \times$ conc. \\
Mgcl $_{2}, 25 \mathrm{mM}$ & $1.6 \mu \mathrm{l}$ & $2 \mathrm{mM}$ \\
Primer mix, $20 \times$ conc. $\{4 \mu \mathrm{M}\}$ & $1.0 \mu \mathrm{l}$ & $0.2 \mu \mathrm{M}$ \\
Template DNA & $5.0 \mu \mathrm{l}$ & $5-30 \mathrm{ng}$ \\
Water, PCR-grade & $2.4 \mu \mathrm{l}$ & - \\
Total volume & $20 \mu \mathrm{l}$ & - \\
\hline
\end{tabular}

number of reactions to be run. Fifteen $\mu \mathrm{l}$ of the PCR mixture was transferred into each well of the LightCycler 480 Instrument multi-well plate (Roche, Germany) then $5 \mu$ l of the DNA template was added, mixed by pipetting up and down. The LightCycler 480 foil was used to seal the multi-well plate before loading it into the LightCycler 480 (Roche, Germany) (Table 3).

A non-template control containing sterile distilled water was included in the experiment. M. tuberculosis wild type strain American Type Culture Collection (ATCC H37) as well as a $M$. bovis strain served as negative and positive controls for the pncA mutation respectively. Collected data were analysed following the final step using Light Cycler 480 software version 1.5. Temperature plots were generated by converting the wild type melting profile to a horizontal line and normalizing the melting profiles of the examined isolates against the $M$. tuberculosis wild-type profile. $M$. tuberculosis isolates with mutations in the pncA sequence were distinguished from the wild type based on the difference in melting temperature observed. At the end of the analysis, the software either reported the isolate as a wild type (PZA susceptible) or mutant (PZA resistant).

\subsection{DNA Sequencing of the pncA Gene of Discordant M. tuberculosis Isolates}

The entire pncA gene was sequenced using two primers, $\mathrm{P} 1$ and $\mathrm{P} 6$, which flank the entire pncA gene and its upstream promoter (700-bp). Purified DNA (10 $\mu$ l) from each isolate was sent for DNA sequencing which was carried out by a commercial sequencing centre using standard Sanger sequencing (InqabaBiotec, SA). Retrieved sequences were compared with the wild type pncA sequence from $M$. tuberculosis $\mathrm{H} 37 \mathrm{Rv}$ for the detection of mutations associated with PZA resistance. Sequence alignment and analysis was performed using special software (CLC Genomics workbench, Denmark). Isolates were either reported as wild type (PZA susceptible) or mutant (PZA resistant). These results were compared to the HRM results, as well as the phenotypic drug susceptibility results obtained from the Bactec MGIT 960 to assess the HRM against a molecular gold standard as well.

\subsection{Statistical Analysis}

Statistical analysis was performed using the Bactec MGIT 960 as a phenotypic gold 
Table 3. PCR amplification and HRM conditions.

\begin{tabular}{cccc}
\hline Steps in the PCR cycle & Temperature & Time & No. of cycles \\
\hline $\begin{array}{c}\text { Initial denaturation } \\
\text { (FastStartTaq DNA Polymerase) }\end{array}$ & $95^{\circ} \mathrm{C}$ & $10: 00 \mathrm{~min}$ & 1 \\
Denaturation & $95^{\circ} \mathrm{C}$ & $00: 10 \mathrm{sec}$ & 45 \\
Annealing & $63^{\circ} \mathrm{C}$ & $15: 00 \mathrm{~min}$ & \\
Extension & $72^{\circ} \mathrm{C}$ & $00: 16 \mathrm{sec}$ \\
& $\mathrm{For}$ HRM & $1: 00 \mathrm{~min}$ \\
& $95^{\circ} \mathrm{C}$ & $00: 01 \mathrm{~min}$ \\
Second hold & $40^{\circ} \mathrm{C}$ & $00: 01 \mathrm{sec}$ \\
& $65^{\circ} \mathrm{C}$ & Fluorescent detection & \\
& $95^{\circ} \mathrm{C}$ & $\left(25\right.$ acquisition per $\left.1^{\circ} \mathrm{C}\right)$ & \\
Second temperature increase & $40^{\circ} \mathrm{C}$ & $00: 10 \mathrm{sec}$ \\
Cooling down & &
\end{tabular}

standard method and DNA sequencing as a molecular gold standard method. Results of HRM and MGIT or sequencing susceptibility testing were entered into $2 \times 2$ tables to calculate the sensitivity (ability to detect true resistance) and specificity (ability to detect true susceptibility) of HRM against MGIT as well as the positive predictive values (PPV) and the negative predictive values (NPV).

\section{Results}

A total of 120 M. tuberculosis isolates were collected from the NHLS-TB Laboratory Tshwane Academic Division (Diagnostic division of the Department of Medical Microbiology, University of Pretoria. Seven isolates were excluded due to duplication. Additional 18 isolates had no HRM results and were excluded from analysis. Susceptibility of the 95 M. tuberculosis isolates included in the final analysis to the first-line anti-TB drugs are shown in Figure 1. Isolates were categorized as MDR-TB (Resistant to both RIF and INH) and Non-MDR-TB (susceptible to at least RIF or/and INH).

\subsection{The BACTEC MGIT 960}

According to the Bactec MGIT 960, 30.5\% (29/95) of the isolates were phenotypically resistant to PZA. Of these 79.3\% (23/29) were MDR and 20.7\% (6/29) were non-MDR. Prazinamide susceptible isolates were detected in 69.4\% (66/95) of the isolates, in which $25.8 \%$ (17/66) were MDR and 74.2\% (49/66) were non-MDR (Table 4). Of the 34 isolates that were phenotypically susceptible to other drugs, 33 were determined to be susceptible to PZA. However, one isolate, which was phenotypically susceptible to other drugs, was PZA-resistant, suggesting PZA mono-resistance (Table 4). The average time of reporting PZA susceptibility results was 8.5 days and $86.3 \%$ of isolates were reported in four to 12 days. 


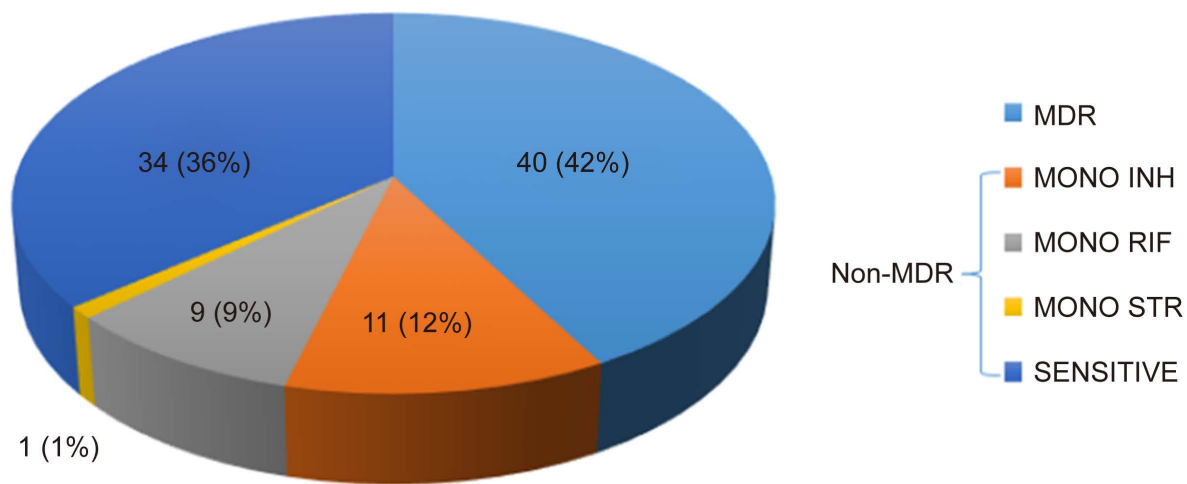

Figure 1. First-line susceptibility profile of $95 \mathrm{M}$. tuberculosis isolates included in the study. MDR = Multi-drug resistant (Resistant to at least RIF and INH); Mono INH = Resistant to INH only; Mono RIF = Resistant to RIF only; Mono STR = Resistant to STR only; Sensitive = Susceptible to both RIF and INH.

Table 4. PZA susceptibility results by the BACTEC MGIT 960.

\begin{tabular}{ccc}
\hline & \multicolumn{3}{c}{ BACTEC MGIT 960 } \\
\cline { 2 - 3 } M. tuberculosis Isolates & R & S \\
MDR-TB $(\boldsymbol{n}=\mathbf{4 0})$ & 23 & 17 \\
Non-MDR-TB $(\boldsymbol{n}=\mathbf{5 5})$ & 6 & 49 \\
Mono Rif $(\boldsymbol{n}=9)$ & 1 & 8 \\
Mono INH $(\boldsymbol{n}=11)$ & 3 & 8 \\
Mono STR $(\boldsymbol{n}=\mathbf{1})$ & 1 & 0 \\
Sensitive $(\boldsymbol{n}=34)$ & 1 & 33
\end{tabular}

MGIT = Mycobacterial Growth Indicator Tube; MDR-TB = Multi-drug resistant tuberculosis; $\mathrm{R}=$ Resistant; $\mathrm{S}=$ Susceptible; Mono INH = Resistant to INH only; Mono RIF = Resistant to RIF only; Mono STR = Resistant to STR only; Sensitive $=$ Susceptible to both RIF and INH.

\subsection{HRM Analysis}

High Resolution Melting curve analysis of the pncA gene was performed in DNA samples isolated from $95 \mathrm{M}$. tuberculosis isolates using three overlapping fragments. Representative normalized melting curves from HRM analysis are shown in Figure 2(a) and Figure 2(b). In the HRM graphs, each line indicates the melt curve profile for an individual sample. The normalized graph shows melting temperature shifts versus wildtype amplicon indicating lower or higher melting temperature. Based on the differences in the shape of the melting curves in any of the three overlapping fragments, $M$. tuberculosis isolates with mutations could easily be differentiated from the susceptible (wildtype) ones. Wild types are represented by blue lines, whereas mutants are represented by red lines. In each batch, DNA isolated from the susceptible control strain (H37Rv) was included as a reference and shown to be wild type at all times (blue line). Based on HRM analysis, $77.9 \%(74 / 95)$ of the isolates were susceptible to PZA while $22.1 \%$ (21/95) were resistant. 


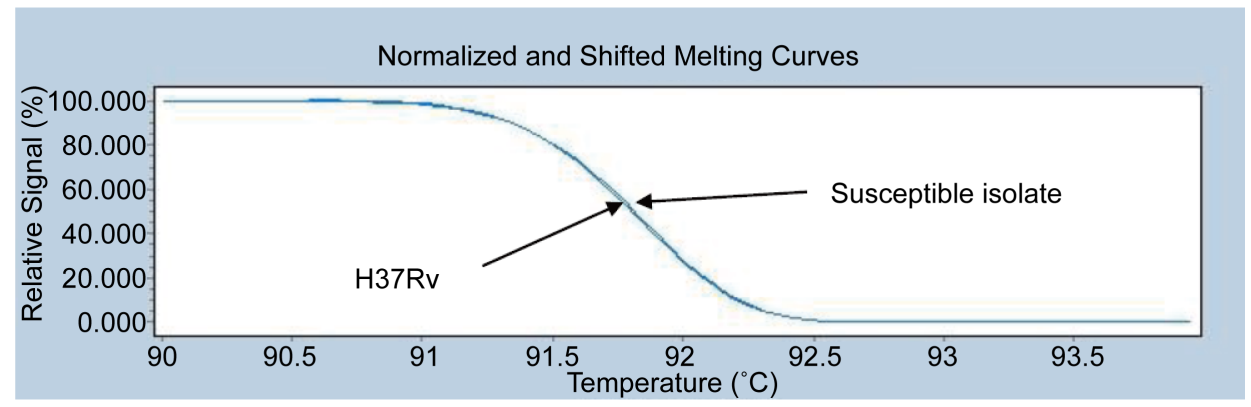

(a)

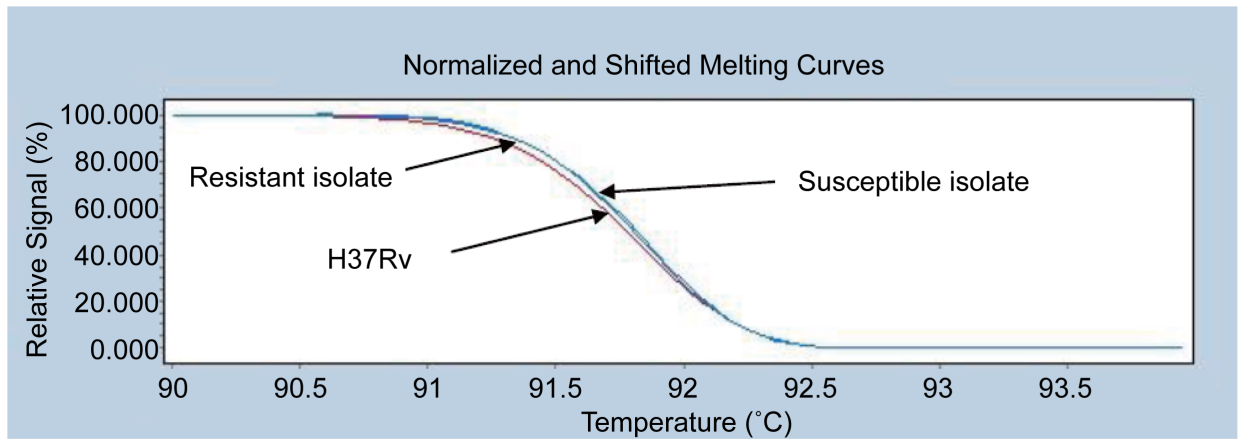

(b)

Figure 2. Normalized melting curves from HRM analysis of the pncA gene. (a): Amplicons amplified from susceptible isolate versus wild-type reference strain (H37Rv); (b): Amplicons amplified from susceptible and resistant isolates versus wild-type reference strain (H37Rv).

\subsection{Comparison of PZA Susceptibility Testing Results for the BACTEC MGIT 960 and HRM Analysis}

Of the 95 isolates included in this study, 55 isolates (57.9\%) and 10 isolates (10.5\%) were have shown resistance and susceptibility by both methods, respectively. Discordant results were observed in 30 isolates (31.6\%). When the Bactec MGIT 960 method was considered as the gold standard, HRM showed a sensitivity (ability to detect true resistance) of $47.7 \%$ and specificity (ability to detect true susceptibility) of $74.6 \%$. The accuracy, the positive and negative predictive value of HRM were $69.5 \%, 34.5 \%$ and $83.3 \%$, respectively. The overall agreement between the two methods was $68.4 \%$ (Table $5)$.

\subsection{DNA Sequencing of pncA Gene}

PCR products were obtained from 48 isolates (50.5\% of the total sample size) and sequenced. These included all isolates that showed resistance to PZA by MGIT 960 (30), all isolates that showed resistance by HRM (11) and seven isolates that were randomly selected from those which were susceptible by both methods i.e. MGIT 960 and HRM (Table 6).

Different types of mutations in the pncA-encoding region were identified in 27 isolates (56.3\%), including nucleotide substitutions, insertions and deletions. No multiplesite mutation was detected (Table 6). Substitution of a single nucleotide was the most 
Table 5. Comparison of PZA susceptibility testing results for the BACTEC MGIT 960 and HRM curve analysis.

\begin{tabular}{cccc}
\hline Test & MGIT 960 & HRM & Number of isolates with results \\
\hline Results for indicated test & S & S & R \\
R & S & R & 10 \\
R & S & 11
\end{tabular}

Performance parameters:

Sensitivity $=47.7 \%$

Specificity $=74.6 \%$

Accuracy $=69.5 \%$

$\mathrm{PPV}=34.5 \%$

$\mathrm{NPV}=83.3 \%$

MGIT = Mycobacterial Growth Indicator Tube; HRM: High Resolution Melting; S = Susceptible, R = Resistant; PPV: Positive predictive value; NPV: Negative predictive value.

Table 6. Drug susceptibility patterns and DNA sequencing of pncA gene of 48 M. tuberculosis isolates.

\begin{tabular}{|c|c|c|c|c|c|c|c|c|}
\hline \multirow{3}{*}{$\begin{array}{l}\text { Isolate } \\
\text { Number }\end{array}$} & \multirow{3}{*}{ Resistance Pattern } & \multicolumn{2}{|c|}{ PZA susceptibility testing } & \multicolumn{5}{|c|}{ DNA Sequencing of pncA gene } \\
\hline & & \multirow{2}{*}{ MGIT } & \multirow{2}{*}{ HRM (F) } & \multirow{2}{*}{$\begin{array}{l}\text { Type of } \\
\text { change }\end{array}$} & \multirow{2}{*}{$\begin{array}{l}\text { Codon } \\
\text { number }\end{array}$} & \multicolumn{2}{|c|}{ Nucleotide change } & \multirow{2}{*}{ Amino acid change } \\
\hline & & & & & & From & To & \\
\hline 1 & RIF, INH,STR, EMB, ETH, KAN, OFX & $\mathrm{R}$ & S & Insertion & 173 & - & $\mathrm{C}$ & Glu173Frameshift ** \\
\hline 2 & INH & S & $\mathrm{R}(\mathrm{F} 3)$ & No change & - & - & - & - \\
\hline 4 & RIF, INH, OFX & $\mathrm{R}$ & $S$ & Substitution & 14 & A & G & Cys14Arg * \\
\hline 5 & IHN, STR & $\mathrm{R}$ & S & Substitution & 51 & G & $\mathrm{C}$ & His51Asp ** \\
\hline 6 & INH, STR, EMB & $\mathrm{R}$ & $\mathrm{R}(\mathrm{F} 1)$ & No change & - & - & - & - \\
\hline 9 & RIF, INH & $\mathrm{R}$ & S & Substitution & 14 & G & $\mathrm{C}$ & Cys14Trp ** \\
\hline 10 & RIF, INH & S & $\mathrm{R}(\mathrm{F} 1)$ & No change & - & - & - & - \\
\hline 11 & RIF, INH & $\mathrm{R}$ & $\mathrm{R}(\mathrm{F} 2, \mathrm{~F} 3)$ & Substitution & 14 & G & $\mathrm{C}$ & Cys14Trp ${ }^{* *}$ \\
\hline 12 & INH & $\mathrm{R}$ & $\mathrm{R}(\mathrm{F} 3)$ & No change & - & - & - & - \\
\hline 13 & RIF, INH & S & S & No change & - & - & - & - \\
\hline 14 & RIF, INH & $\mathrm{R}$ & S & Substitution & 134 & G & $\mathrm{T}$ & Ala134Asp ${ }^{\star *}$ \\
\hline 15 & RIF, INH & S & $\mathrm{R}(\mathrm{F} 3)$ & Substitution & 139 & A & $\mathrm{C}$ & Val139Gly* \\
\hline
\end{tabular}


Continued

\begin{tabular}{|c|c|c|c|c|c|c|c|c|}
\hline 20 & RIF, INH & $\mathrm{R}$ & $\mathrm{R}(\mathrm{F} 1, \mathrm{~F} 2, \mathrm{~F} 3)$ & Deletion & 21 & A & - & Val21Frameshift ${ }^{* *}$ \\
\hline 21 & RIF, INH OFX & $\mathrm{S}$ & S & Substitution & 10 & G & A & Ala10Val * \\
\hline 22 & RIF, INH, EMB & $\mathrm{R}$ & $\mathrm{R}(\mathrm{F} 1)$ & Substitution & 14 & $\mathrm{~A}$ & G & Cys14Arg * \\
\hline 23 & RIF, INH & $S$ & $S$ & Substitution & 14 & G & $\mathrm{C}$ & Cys14Trp ** \\
\hline 24 & STR & $\mathrm{R}$ & $S$ & No change & - & - & - & - \\
\hline 25 & RIF, INH & $\mathrm{R}$ & $S$ & No change & - & - & - & - \\
\hline 26 & RIF, INH & $S$ & S & No change & - & - & - & - \\
\hline 27 & RIF & $\mathrm{R}$ & $S$ & No change & - & - & - & - \\
\hline 28 & RIF, INH, STR, EMB & $\mathrm{R}$ & $S$ & Substitution & 135 & $\mathrm{~T}$ & G & Thr135Pro * \\
\hline 29 & RIF, INH & $S$ & $\mathrm{R}(\mathrm{F} 3)$ & Substitution & 139 & $\mathrm{~A}$ & $\mathrm{C}$ & Val139Gly* \\
\hline 30 & RIF & $S$ & $\mathrm{R}(\mathrm{F} 1)$ & Substitution & 172 & A & G & Leu172Pro * \\
\hline 31 & RIF, INH & $\mathrm{R}$ & $\mathrm{R}(\mathrm{F} 2)$ & Substitution & 59 & $\mathrm{~A}$ & G & Ser59Pro ** \\
\hline 32 & RIF, INH & $\mathrm{R}$ & S & Substitution & 135 & $\mathrm{~T}$ & G & Thr135Pro * \\
\hline 33 & RIF, INH & $\mathrm{R}$ & $S$ & Substitution & 135 & $\mathrm{~T}$ & G & Thr135Pro * \\
\hline 34 & RIF, INH, EMB & $\mathrm{R}$ & $S$ & Substitution & 135 & $\mathrm{~T}$ & G & Thr135Pro * \\
\hline 35 & RIF, INH, STR & $\mathrm{R}$ & S & Substitution & 135 & $\mathrm{~T}$ & G & Thr135Pro * \\
\hline 36 & RIF, INH & $\mathrm{R}$ & S & No change & - & - & - & - \\
\hline 37 & RIF, INH & $\mathrm{R}$ & S & Insertion & 12 & - & $\mathrm{C}$ & Asp12Frameshift ** \\
\hline 38 & Susceptible & $S$ & $\mathrm{R}(\mathrm{F} 1, \mathrm{~F} 3)$ & Substitution & 122 & G & $\mathrm{A}$ & Gln122Stop ** \\
\hline 39 & RIF, INH & $\mathrm{R}$ & S & Substitution & 12 & $\mathrm{~T}$ & $\mathrm{C}$ & Asp12Gly * \\
\hline 40 & RIF, INH & $\mathrm{R}$ & $S$ & No change & - & - & - & - \\
\hline 41 & Susceptible & $\mathrm{S}$ & $S$ & No change & - & - & - & - \\
\hline 42 & Susceptible & $S$ & $\mathrm{R}(\mathrm{F} 3)$ & No change & - & - & - & - \\
\hline 43 & Susceptible & $\mathrm{S}$ & S & No change & - & - & - & - \\
\hline 44 & Susceptible & $\mathrm{S}$ & $S$ & No change & - & - & - & - \\
\hline 45 & Susceptible & $\mathrm{S}$ & $S$ & No change & - & - & - & - \\
\hline 46 & Susceptible & $\mathrm{R}$ & S & Substitution & 57 & G & $\mathrm{C}$ & His57Asp ** \\
\hline 47 & Susceptible & $\mathrm{S}$ & $\mathrm{R}(\mathrm{F} 1, \mathrm{~F} 2, \mathrm{~F} 3)$ & No change & - & - & - & - \\
\hline 48 & Susceptible & $\mathrm{S}$ & $\mathrm{R}(\mathrm{F} 2, \mathrm{~F} 3)$ & No change & - & - & - & - \\
\hline $\mathrm{H} 37 \mathrm{Rv}$ & Susceptible & $\mathrm{S}$ & $S$ & No change & - & - & - & - \\
\hline
\end{tabular}

PZA = Pyrazinamide; MGIT = Mycobacterial Growth Indicator Tube; HRM: High Resolution Melting; (F): Fragment showing variation by HRM; RIF = Rifampicin; INH = Isoniazid; STR = Streptomycin; EMB = Ethambutol; ETH = Ethionamide; KAN = Kanamycin; OFX = Ofloxacin; S = Susceptible; R = Resistant; A = Adenine; T = Thymine; $\mathrm{C}$ = Cytosine; $\mathrm{G}=$ Guanine; H37Rv = Control strain (Wild-type); ( $\mathrm{a}$ ) = Number of codon position was counted from the start codon (ATG) of the pncA; $\left({ }^{*}\right)$ = High confidence mutations (mutations associated with in-vitro documented resistance reported by at least 10 publications); $\left({ }^{*}\right)=$ New mutations (reported for the first time). 
common mutation $88.9 \%$ (24/27), followed by insertion $7.4 \%(2 / 27)$ and deletion $3.7 \%$ $(1 / 27)$. No mutation was detected in $21(43.7 \%)$ isolates, of these, four (19\%) were resistant by both methods, five (23.8\%) were resistant by MGIT 960 only, five (23.8\%) were resistant by HRM only and seven (33.4\%) were susceptible by both methods (Table 6).

As indicated in Table 6, the mutation rate among the phenotypically resistant isolates that were reported by MGIT 960 was $65.5 \%$ (19/29), whereas mutations were only detected in $45.5 \%$ (5/11) isolates that were initially reported as resistant by HRM. Additionally, mutations were shown in $60 \%(6 / 10)$ of the isolates that were reported resistant by both methods. No mutation was identified in the wild-type (H37Rv) strain. However, two isolates, despite being susceptible by both MGIT and HRM, showed mutations in the pncA gene. High confidence mutations, i.e. mutations associated with in-vitro documented resistance reported by at least 10 publications were found in $51.8 \%(14 / 27)$ of isolates. The rest, $48.2 \%$ (13/27), were new mutations which have not been reported in previous studies.

The most frequently mutated sites were at codon 135 , which showed a substitution from thymine to guanine at nucleotide 403 , this resulted in a switch at translation from threonine to proline in five isolates (18.5\%). Another common site was codon 139 which showed a substitution from adenine to cytosine at nucleotide 416 , and resulted in a switch at translation from valine to glycine in three isolates (11.1\%). Additional three isolates $(11.1 \%)$ showed new mutations in codon 14, switching translation from cysteine to tryptophan. These three mutation sites accounted for more than one third of all the mutations identified (Table 6).

\subsection{Comparison of HRM Analysis and the BACTEC MGIT 960 with DNA Sequencing}

Isolates with discordant results between HRM analysis and the Bactec MGIT PZA susceptibility were compared with DNA sequencing of the pncA gene (Table 7). HRM analysis was concordant with DNA sequencing for $47.9 \%(23 / 48)$ of the isolates. Among the 25 discordant isolates, 16 isolates had mutations in the pncA gene while HRM detected no variation; and 9 had no mutations in the pncA while HRM detected variation. DNA Sequencing of pncA was concordant with the Bactec MGIT 960 PZA susceptibility testing for $66.7 \%(32 / 48)$ of the isolates. Among the 16 discordant isolates, nine were PZA resistant while sequencing detected no mutation, and seven were PZA susceptible while sequencing showed mutations. Overall, there were fewer discrepancies with MGIT than with HRM.

\section{Discussion}

Although PZA forms an integral part of both first line and MDR TB management, routine susceptibility testing of this drug is not performed due to various challenges present in the currently available techniques. This study evaluated the rapid technique of HRM for the detection of PZA resistance against the phenotypic gold standard (Bactec 
Table 7. Comparison of HRM analysis and The BACTEC MGIT 960 with DNA sequencing.

\begin{tabular}{|c|c|c|c|c|c|}
\hline DNA Sequencing & \multicolumn{2}{|c|}{ HRM PZA Susceptibility $(n=48)$} & DNA Sequencing & \multicolumn{2}{|c|}{ MGIT PZA Susceptibility $(n=48)$} \\
\hline Mutation & 16 & 11 & Mutation & 7 & 20 \\
\hline \multirow[t]{5}{*}{ No mutation } & 12 & 9 & No mutation & 12 & 9 \\
\hline & Performance para & & & ance parame & \\
\hline & Accuracy $=47$ & & & aracy $=66.7 \%$ & \\
\hline & $\mathrm{PPV}=55.0^{\circ}$ & & & $\mathrm{V}=74.07 \%$ & \\
\hline & $\mathrm{NPV}=42.86$ & & & $\mathrm{~V}=57.14 \%$ & \\
\hline
\end{tabular}

MGIT = Mycobacterial Growth Indicator Tube; HRM: High Resolution Melting; PZA: Pyrazinamide; PPV: Positive predictive value; NPV: Negative predictive value.

MGIT 960).

In this study, the Bactec MGIT 960 results showed a prevalence of PZA resistance of $57.5 \%(23 / 40)$ among the MDR-TB isolates tested, while the prevalence among nonMDR isolates was $10.9 \%$ (6/55). High rates of PZA resistance among MDR-TB have been observed in previous studies, ranging from $49 \%$ in Thailand [13], to 50\% in Central Africa [14], 52\% in South Africa [15], 53\% in Japan [16] and 55\% in Taiwan [17]. Studies from Pakistan [18], South Korea Kim et al., 2012 [19] and India [20] have shown higher rates of resistance, $77 \%, 85 \%$ and $85 \%$ respectively. These high rates of PZA resistance in $\mathrm{TB}$ endemic countries may be attributed to the widespread use of PZA in re-treatment regimens in these areas, particularly when used without relying on susceptibility testing results. The high occurrence of false resistance by phenotypic susceptibility testing could also cause the overestimation of PZA resistance among MDRTB [21].

In 2013, Nagai et al. developed and evaluated HRM curve analysis for mutation detection in four major anti-TB drugs, namely RIF, INH, EMB, and STR. Their HRM assay was successful in detecting mutations in genes associated with resistance ( $r p o \mathrm{~B}$, $k a t \mathrm{G}, \operatorname{inh} \mathrm{A}, a m b \mathrm{~B}, r p s \mathrm{~L}$ and $r r s$ ) and results were completely consistent with those of DNA sequencing. Sensitivity and specificity of HRM were $100 \%$ and $100 \%$ for RIF, $88.8 \%$ and $100 \%$ for INH, $100 \%$ and $100 \%$ for EMB, and $100 \%$ and $93.7 \%$ for STR, respectively [22].

Comparable results were also achieved by another two recent studies when HRM was used for the detection of PZA resistance. Hong et al. reported a sensitivity and specificity of $85.5 \%$ and $98.5 \%$, respectively. Whereas Pholwat et al. showed concordance of 84\% between HRM and phenotypic PZA susceptibility testing [23] [24]. The agreement between the phenotypic Bactec MGIT 960 and HRM for PZA susceptibility in this study was only $68.4 \%$. Sensitivity and specificity of HRM were $47.7 \%$ and $74.6 \%$, respectively. These values were much lower than previously reported [24] [25] [26]. Discrepancies between the two methods detected on 19 PZA-resistant isolates that were reported wild-type by $\mathrm{HRM}$, and on $10 \mathrm{PZA}$-susceptible isolates that showed mutations by HRM. Among these 19 isolates, sequencing detected mutations in 14 of them sug- 
gesting that HRM was not able to detect these mutants which were also phenotypically resistant. The majority variants were SNPs with five of the 19 occurring at codon 135 .

Conversely, five of the ten susceptible isolates which were reported as variants by HRM showed no mutation on sequencing. Although this would suggest that these were false on HRM, it is also possible that the HRM result is true since HRM is PCR based and has a greater likelihood of detecting mixed populations which may not be detected by Sanger sequencing, and these sub-populations may not be large enough to show resistance phenotypically. We did not perform next generation sequencing which may have provided an answer to this uncertainty.

Recent studies have indicated that the new "gold standard" for PZA resistance determination should be sequencing. When considering the discordant isolates only and comparing HRM and MGIT 960 against this "new" gold standard, the accuracy for HRM was $47.9 \%$ while that of the Bactec MGIT 960 was slightly better at $66.7 \%$ (Table 7).

Phenotypic resistance to PZA has been correlated with mutations in the pncA gene in several previous studies. Some of these studies have reported this correlation to be inconsistent, ranging between $41 \%$ and $80 \%$ [25]-[31], whereas other studies showed higher correlation ranging from 91\% - 97\% [15] [17] [32] [33] [34]. In the current study, based on sequencing results, a correlation of 0.67 (significant at $p$-value $<0.05$ ) between phenotypic resistance to PZA and pncA mutation was observed, this is comparable to majority of the previous studies, more especially to the study that was conducted in South Africa by Bishop et al., in which a correlation of $67 \%$ was also reported [35].

The mutations detected in our study were found to be scattered along the entire pncA gene with no major hot spots identified. However, mutations at codon 135 were found in five isolates, whereas mutations at codons 14 and 139 were shown by three isolates each. These observations of scattered mutations are supported by similar findings in previous studies [27] [36]. The high diversity of pncA mutations limits its inclusion in the current molecular techniques such as GenoType MTBDRplus (Hain Lifescience) [37].

Absence of mutations in the pncA gene or its upper promoter in $26.7 \%$ of the PZAresistant isolates we tested correlated with the study that was done by Sreevatsan et al., in which they reported no pncA mutations in $28 \%$ of PZA-resistant M. tuberculosis strains tested [15]. These cases could also be explained by another mechanism of resistance to PZA. Recently, rpsA gene has been shown to play a role in PZA resistance and has been recommended to be used as an additional target for the molecular detection of PZA susceptibility [9] [38], however in another study, none of the PZA-resistant strains harboured mutations in the rpsA gene [39]. Based on these variations, the association between PZA resistance and rpsA mutations remains un-established and requires further investigations in the future.

The low sensitivity and specificity of HRM in this study could be due to the use of three sets of primers as compared to five or seven as used in previous studies [40] [41]. 
Unlike other studies, the current HRM was developed and evaluated using the Roche LightCycler 480 . So far, only one study has utilized the Roche LightCycler $480^{\circ}$ for PZA susceptibility testing and used the Bactec MGIT 960 as a reference method [42]. They have shown a sensitivity and specificity of $85.5 \%$ and $98.5 \%$, respectively and concluded that HRM is a rapid and accurate test for the detection of PZA resistance and can be used as a screening method [26]. However, recently, HRM results generated from the Rotor-gene system for the detection of PZA resistance have been found to be easier to interpret than other platforms [25].

Another explanation for the low correlation between PZA resistance and pncA mutations in this study is the possibility of phenotypic false resistance. This observation has been proven elsewhere [25]. PZA phenotypic susceptibility testing by the Bactec MGIT 960 is normally carried out at $\mathrm{pH}$ of 5.9, resistance could be falsely reported as a result of the ammonia produced during mycobacterial growth; ammonia elevates the $\mathrm{pH}$ of the media thus inactivating the drug [26]. According to the Henderson-Hasselbalch equation, M. tuberculosis should be tested for PZA susceptibility at a concentration of at least $156 \mu \mathrm{g} / \mathrm{ml}$ [10], rather than the current cut-off used in the Bactec MGIT 960 method $(100 \mu \mathrm{g} / \mathrm{ml})$. PZA resistance could also be caused by the action of efflux pumps which has been shown to play a role in mycobacterial resistance [43]. Detection of pncA mutations in the susceptible isolates suggests that these mutations might not be fully expressed to show phenotypic PZA resistance.

\section{Conclusions}

In this study, apart from simplicity and speed, the current HRM genotypic method to determine PZA susceptibility using the gene scanning software of the Roche LightCycler 480 was shown to be moderately specific with low sensitivity. Further modifications to improve its performance are required. Pyrazinamide resistance was common among MDR-TB and the current study results showed low association of the phenotypic PZA resistance with mutations in the pncA gene. This warrants further studies to determine potential alternate genetic mechanisms of resistance to PZA in our context and reevaluation of the MGIT 960 for detection of phenotypic resistance with an alternate phenotypic method such as the Wayne's test.

This study provides an important baseline for more extensive evaluation studies to improve and validate the use of HRM in determining PZA susceptibility. In the future, using more than three fragments (six or seven) should be considered as this could increase the sensitivity and specificity of HRM in detecting pncA mutations. However, this might also increase the cost of the test. Additionally, combining such data to results from the biochemical analysis of PZase enzyme (Wayne's test) would add to its value and ease the interpretation of the final results.

\section{Conflict of Interest}

The authors declare that they have no financial or non-financial competing interests. 


\section{Funding}

This project was supported by grant from the National Health Laboratory Service of South Africa.

\section{References}

[1] Ahmady, A., Poolad, T., Rafee, P., Tousheh, M., Kahbazi, M. and Arjomandzadegan, M. (2013) Study of Pyrazinamidase Structural Changes in Pyrazinamide Resistant and Susceptible Isolates of Mycobacterium tuberculosis. Tuberk Toraks, 61, 110-114. http://dx.doi.org/10.5578/tt.3888

[2] Yee, D.P., Menzies, D. and Brassard, P. (2012) Clinical Outcomes of Pyrazinamide-Monoresistant Mycobacterium tuberculosis in Quebec. International Journal of Tuberculosis and Lung Disease, 16, 604-609.

[3] Raynaud, C., Lanéelle, M., Senaratne, R.H., Draper, P., Lanéelle, G. and Daffé, M. (1999) Mechanisms of Pyrazinamide Resistance in Mycobacteria: Importance of Lack of Uptake in Addition to Lack of Pyrazinamidase Activity. Microbiology, 145, 1359-1367. http://dx.doi.org/10.1099/13500872-145-6-1359

[4] Sheen, P., Lozano, K., Gilman, R.H., Valencia, H.J., Loli, S., Fuentes, P., et al. (2013) pncA Gene Expression and Prediction Factors on Pyrazinamide Resistance in Mycobacterium tuberculosis. Tuberculosis, 93, 515-522. http://dx.doi.org/10.1016/j.tube.2013.03.005

[5] Zimic, M., Loli, S., Gilman, R.H., Gutierrez, A., Fuentes, P., Cotrina, M., et al. (2012) A New Approach for Pyrazinamide Susceptibility Testing in Mycobacterium tuberculosis. Microbial Drug Resistance, 4, 372-375. http://dx.doi.org/10.1089/mdr.2011.0207

[6] Ajbani, K., Nikam, C., Kazi, M., Gray, C., Boehme, C., Balan, K., et al. (2012) Evaluation of Genotype MTBDRsl Assay to Detect Drug Resistance Associated with Fluoroquinolones, Aminoglycosides and Ethambutol on Clinical Sediments. PLoS One, 7, e49433. http://dx.doi.org/10.1371/journal.pone.0049433

[7] Jureen, P., Werngren, J. and Hoffner, S.E. (2004) Evaluation of the Line Probe Assay (LiPA) for Rapid Detection of Rifampicin Resistance in Mycobacterium tuberculosis. Tuberculosis, 84, 311-316. http://dx.doi.org/10.1016/j.tube.2003.12.001

[8] Morlock, G.P., Crawford, J.T., Butler, W.R., Brim, S.E., Sikes, D., Mazurek, G.H., et al. (2000) Phenotypic Characterization of pncA Mutants of Mycobacterium tuberculosis. Antimicrobial Agents and Chemotherapy, 44, 2291-2295. http://dx.doi.org/10.1128/AAC.44.9.2291-2295.2000

[9] Sreevatsan, S., Pan, X., Zhang, Y., Kreiswirth, B.N. and Musser, J.M. (1997) Mutations Associated with Pyrazinamide Resistance in pncA of Mycobacterium tuberculosis Complex Organisms. Antimicrobial Agents and Chemotherapy, 41, 636-640.

[10] Scorpio, A., Lindholm-Levy, P., Heifets, L., Gilman, R., Siddiqi, S., Cynamon, M., et al. (1997) Characterization of pncA Mutations in Pyrazinamide-Resistant Mycobacterium tuberculosis. Antimicrobial Agents and Chemotherapy, 41, 540-543.

[11] Slany, M., Vanerkova, M., Nemcova, E., Zaloudikova, B., Ruzicka, F. and Freiberger, T. (2010) Differentiation of Staphylococcus spp. by High-Resolution Melting Analysis. Canadian Journal of Microbiology, 56, 1040-1049. http://dx.doi.org/10.1139/W10-091

[12] Merchant-Patel, S., Blackall, P.J., Templeton, J., Price, E.P., Tong, S.Y., Huygens, F., et al. (2010) Campylobacter jejuni and Campylobacter coli Genotyping by High-Resolution Melting Analysis of a flaA Fragment. Applied and Environmental Microbiology, 76, 493499. http://dx.doi.org/10.1128/AEM.01164-09 
[13] Choi, G.E., Lee, S.M., Yi, J., Hwang, S.H., Kim, H.H., Lee, E.Y., et al. (2010) High- Resolution Melting Curve Analysis for Rapid Detection of Rifampin and Isoniazid Resistance in Mycobacterium tuberculosis Clinical Isolates. Journal of Clinical Microbiology, 48, 38933898. http://dx.doi.org/10.1128/JCM.00396-10

[14] Scorpio, A. and Zhang, Y. (1996) Mutations in pncA, a Gene Encoding Pyrazinamidase/ Nicotinamidase, Cause Resistance to the Antituberculous Drug Pyrazinamide in Tubercle Bacillus. Nature Medicine, 2, 662-667. http://dx.doi.org/10.1038/nm0696-662

[15] Jonmalung, J., Prammananan, T., Leechawengwongs, M. and Chaiprasert, A. (2010) Surveillance of Pyrazinamide Susceptibility among Multidrug-Resistant Mycobacterium tuberculosis Isolates from Siriraj Hospital, Thailand. BMC Microbiology, 10, 223. http://dx.doi.org/10.1186/1471-2180-10-223

[16] Minime-Lingoupou, F., Pierre-Audigier, C., Kassa-Kelembho, E., Barilone, N., Zandanga, G., Rauzier, J., et al. (2010) Rapid Identification of Multidrug-Resistant Tuberculosis Isolates in Treatment Failure or Relapse Patients in Bangui, Central African Republic. International Journal of Tuberculosis and Lung Disease, 14, 782-785.

[17] Louw, G.E., Warren, R.M., Donald, P.R., Murray, M.B., Bosman, M., Van Helden, P.D., et al. (2006) Frequency and Implications of Pyrazinamide Resistance in Managing Previously Treated Tuberculosis Patients. International Journal of Tuberculosis and Lung Disease, 10, 802-807.

[18] Ando, H., Mitarai, S., Kondo, Y., Suetake, T., Sekiguchi, J.I., Kato, S., et al. (2010) Pyrazinamide Resistance in Multidrug-Resistant Mycobacterium tuberculosis Isolates in Japan. Clinical Microbiology and Infection, 16, 1164-1168. http://dx.doi.org/10.1111/j.1469-0691.2009.03078.x

[19] Chiu, Y.C., Huang, S.F., Yu, K.W., Lee, Y.C., Feng, J.Y. and Su, W.J. (2011) Characteristics of pncA Mutations in Multidrug-Resistant Tuberculosis in Taiwan. BMC Infectious Diseases, $11,240$.

[20] Rao, N.A., Irfan, M., Soomro, M.M. and Mehfooz, Z. (2010) Drug Resistance Pattern in Multidrug Resistance Pulmonary Tuberculosis Patients. Journal of the College of Physicians and Surgeons Pakistan, 20, 262-265.

[21] Kim, H.J., Kwak, H.K., Lee, J., Yun, Y.J., Lee, J.S., Lee, M.S., et al. (2012) Patterns of pncA Mutations in Drug-Resistant Mycobacterium tuberculosis Isolated from Patients in South Korea. International Journal of Tuberculosis and Lung Disease, 16, 98-103. http://dx.doi.org/10.5588/ijtld.10.0739

[22] Shenai, S., Rodrigues, C., Sadani, M., Sukhadia, N. and Mehta, A. (2009) Comparison of Phenotypic and Genotypic Methods for Pyrazinamide Susceptibility Testing. Indian Journal of Tuberculosis, 56, 82-90.

[23] Zhang, Y., Chiu Chang, K., Leung, C., Wai Yew, W., Gicquel, B., Fallows, D., et al. (2012) “ $Z^{S}$-MDR-TB" versus " $Z$ R-MDR-TB": Improving Treatment of MDR-TB by Identifying Pyrazinamide Susceptibility. Emerging Microbes \& Infections, 1, e5. http://dx.doi.org/10.1038/emi.2012.18

[24] Nagai, Y., Iwade, Y., Hayakawa, E., Nakano, M., Sakai, T., Mitarai, S., et al. (2013) High Resolution Melting Curve Assay for Rapid Detection of Drug-Resistant Mycobacterium tuberculosis. Journal of Infection and Chemotherapy, 19, 1116-1125. http://dx.doi.org/10.1007/s10156-013-0636-3

[25] Hong, C.Y., Wang, F. and Liu, X.L. (2013) Detection of pncA Mutation Associated with Pyrazinamide Resistance in Mycobacterium tuberculosis by High-Resolution Melting Curve Analysis. Chinese Journal of Tuberculosis and Respiratory Diseases, 36, 198-201.

[26] Pholwat, S., Stroup, S., Gratz, J., Trangan, V., Foongladda, S., Kumburu, H., et al. (2014) 
Pyrazinamide Susceptibility Testing of Mycobacterium tuberculosis by High Resolution Melt Analysis. Tuberculosis, 94, 20-25. http://dx.doi.org/10.1016/j.tube.2013.10.006

[27] Bishop, K.S., Blumberg, L., Trollip, A.P., Smith, A.N., Roux, L., York, D.F., et al. (2001) Characterisation of the pncA Gene in Mycobacterium tuberculosis Isolates from Gauteng, South Africa. International Journal of Tuberculosis and Lung Disease, 5, 952-957.

[28] Huang, T.S., Lee, S.S., Tu, H.Z., Huang, W.K., Chen, Y.S., Huang, C.K., et al. (2003) Correlation between Pyrazinamide Activity and pncA Mutations in Mycobacterium tuberculosis Isolates in Taiwan. Antimicrobial Agents and Chemotherapy, 47, 3672-3673. http://dx.doi.org/10.1128/AAC.47.11.3672-3673.2003

[29] Miyagi, C., Yamane, N., Yogesh, B., Ano, H. and Takashima, T. (2004) Genetic and Phenotypic Characterization of Pyrazinamide-Resistant Mycobacterium tuberculosis Complex Isolates in Japan. Diagnostic Microbiology and Infectious Disease, 48, 111-116. http://dx.doi.org/10.1016/j.diagmicrobio.2003.09.013

[30] Rodrigues Vde, F., Telles, M.A., Ribeiro, M.O., Cafrune, P.I., Rossetti, M.L. and Zaha, A. (2005) Characterization of pncA Mutations in Pyrazinamide-Resistant Mycobacterium tuberculosis in Brazil. Antimicrobial Agents and Chemotherapy, 49, 444-446. http://dx.doi.org/10.1128/AAC.49.1.444-446.2005

[31] Chan, R.C.Y., Hui, M., Chan, E.W.C., Au, T.K., Chin, M.L., Yip, C.K., et al. (2007) Genetic and Phenotypic Characterization of Drug-Resistant Mycobacterium tuberculosis Isolates in Hong Kong. Journal of Antimicrobial Chemotherapy, 59, 866-873. http://dx.doi.org/10.1093/jac/dkm054

[32] Pandey, S., Newton, S., Upton, A., Roberts, S. and Drinković, D. (2009) Characterisation of pncA Mutations in Clinical Mycobacterium tuberculosis Isolates in New Zealand. Pathology, 41, 582-584. http://dx.doi.org/10.1080/00313020903071587

[33] Hou, L., Osei-Hyiaman, D., Zhang, Z., Wang, B., Yang, A. and Kano, K. (2000) Molecular Characterization of pncA Gene Mutations in Mycobacterium tuberculosis Clinical Isolates from China. Epidemiology and Infection, 124, 227-232. http://dx.doi.org/10.1017/S0950268899003635

[34] Mphahlele, M., Syre, H., Valvatne, H., Stavrum, R., Mannsåker, T., Muthivhi, T., et al. (2008) Pyrazinamide Resistance among South African Multidrug-Resistant Mycobacterium tuberculosis Isolates. Journal of Clinical Microbiology, 46, 3459-3464. http://dx.doi.org/10.1128/JCM.00973-08

[35] Hirano, K., Takahashi, M., Kazumi, Y., Fukasawa, Y. and Abe, C. (1997) Mutation in pncA Is a Major Mechanism of Pyrazinamide Resistance in Mycobacterium tuberculosis. Tubercle and Lung Disease, 78, 117-122. http://dx.doi.org/10.1016/S0962-8479(98)80004-X

[36] Lee, K.W., Lee, J.M. and Jung, K.S. (2001) Characterization of pncA Mutations of Pyrazinamide-Resistant Mycobacterium tuberculosis in Korea. Journal of Korean Medical Science, 16, 537-543. http://dx.doi.org/10.3346/jkms.2001.16.5.537

[37] Doustdar, F., Khosravi, A.D. and Farnia, P. (2009) Mycobacterium tuberculosis Genotypic Diversity in Pyrazinamide-Resistant Isolates of Iran. Microbial Drug Resistance, 15, 251 256. http://dx.doi.org/10.1089/mdr.2009.0066

[38] Hillemann, D., Rusch-Gerdes, S. and Richter, E. (2007) Evaluation of the GenoType MTBDRplus Assay for Rifampin and Isoniazid Susceptibility Testing of Mycobacterium tuberculosis Strains and Clinical Specimens. Journal of Clinical Microbiology, 45, 26352640. http://dx.doi.org/10.1128/JCM.00521-07

[39] Shi, W., Zhang, X., Jiang, X., Yuan, H., Lee, J.S., Barry, C.E., et al. (2011) Pyrazinamide Inhibits Trans-Translation in Mycobacterium tuberculosis. Science, 333, 1630-1632. 
http://dx.doi.org/10.1126/science.1208813

[40] Tan, Y., Hu, Z., Zhang, T., Cai, X., Kuang, H., Liu, Y., et al. (2014) Role of pncA and rpsA Gene Sequencing in Detection of Pyrazinamide Resistance in Mycobacterium tuberculosis Isolates from Southern China. Journal of Clinical Microbiology, 52, 291-297.

http://dx.doi.org/10.1128/JCM.01903-13

[41] Bhuju, S., Fonseca, L.D., Marsico, A.G., de Oliveira Vieira, G.B., Sobral, L.F., Stehr, M., et al. (2013) Mycobacterium tuberculosis Isolates from Rio de Janeiro Reveal Unusually Low Correlation between Pyrazinamide Resistance and Mutations in the pncA Gene. Infection Genetics and Evolution, 19, 1-6. http://dx.doi.org/10.1016/j.meegid.2013.06.008

[42] Watcharasamphankul, W., Houpt, E.R. and Foongladda, S. (2013) Rapid Detection of Pyrazinamide Resistant Mycobacterium tuberculosis by High Resolution Melting Curve Analysis. Journal of the Medical Association of Thailand, 96, 1218-1223.

[43] Zhang, Y., Permar, S. and Sun, Z. (2002) Conditions That May Affect the Results of Susceptibility Testing of Mycobacterium tuberculosis to Pyrazinamide. Journal of Medical Microbiology, 51, 42-49. http://dx.doi.org/10.1099/0022-1317-51-1-42

Submit or recommend next manuscript to SCIRP and we will provide best service for you:

Accepting pre-submission inquiries through Email, Facebook, LinkedIn, Twitter, etc. A wide selection of journals (inclusive of 9 subjects, more than 200 journals)

Providing 24-hour high-quality service

User-friendly online submission system

Fair and swift peer-review system

Efficient typesetting and proofreading procedure

Display of the result of downloads and visits, as well as the number of cited articles Maximum dissemination of your research work

Submit your manuscript at: http://papersubmission.scirp.org/

Or contact jtr@scirp.org 